\title{
DESIGN OF A MULTI-RETAILER SINGLE PRODUCT DISTRIBUTION SYSTEM
}

\author{
K. Pitsa, University of Piraeus, Greece \\ S. Sofianopoulou, University of Piraeus, Greece
}

\begin{abstract}
Over the last decade there has been a growing amount of interest towards the integration of various elements of the Supply Chain Management. The category of problems arising from the combination of distribution and inventory management decisions is possibly the most typical example of that notion and is known as inventory routing problems (IRP). IRP for a single product, deals with the distribution of the product, from a single depot to a set of n customers over a specific number of time periods, using a fleet of homogenous vehicles. The objective is to minimize the average daily cost for the planning period, while ensuring that no stockouts will occur. In this work a two-phase approach is examined. The epicentre of the first phase is a Mathematical Programming model used to provide an accurate description of the problem. At the second phase a Genetic Algorithm (GA) with suitably designed genetic operators, will be employed in order to obtain near optimal solutions.
\end{abstract}

\section{INTRODUCTION}

Over the last decade there has been a growing amount of interest towards the combination-integration of various elements of the Supply Chain Management. As the business world comes to realize the potential this has to offer, i.e. significant savings in inventory handling cost, increased efficiency, higher delivery performance to requested or committed due dates, more researchers are focusing on implementing techniques that render this integration not only viable but also profitable. The present work focuses on one of the most typical examples of this concept, that is, the integration of distribution management and inventory control. Specifically, instead of the customers having to monitor their inventory and make sure that it is replenished on a regular basis to prevent stock-outs, the supplier is responsible for serving all customer needs by determining simultaneously the timing and sizes of the deliveries as well as efficient vehicle schedules so as to minimize total transportation and inventory carrying costs. The category of problems arising from the combination of distribution and inventory management decisions is known as "Inventory Routing Problems" (IRP) and generally is considered to be an extension of the Vehicle Routing Problems (VRP). A variety of industries have provided fertile ground for the development of IRP applications e.g. industrial gases companies, automotive companies etc. fuelling the interest of researchers that have generated numerous methods of solving IRP. Most of these methods propose either a "theoretical" approach or a more "practical" one. The theoretical one, consists of attempting to specify the lower bounds to the problem, whereas the practical one employs heuristics in order to obtain near optimal solutions.

In this paper a multi-retailer single product routing problem is examined and thoroughly reviewed. In the following section the problem is formally stated, while in the next section Genetic Algorithms are introduced and related operators are discussed. Then, the application of the algorithmic procedure to IRP is implemented, while concluding remarks and suggestions for further research are presented in the last section.

In order to categorise the approaches developed for the solution of IRP, we will adopt the classification introduced by Bramel and Simchi-Levi (1997). According to this, most of the research on IRP is in one of three directions: single-day models using deterministic demand (Chien, Balakrishnan, and Wong, 1989; Bertazzi, Paletta, and Speranza, 2002), multi-day models using deterministic demand (Bard, Huang, Jaillet and Dror 1998; Campbell and Savelsbergh 2004), and infinite time horizon usually for long-term planning purposes (Anily and Bramel, 2004 ). Finally stochastic demand has also been considered by several researchers (Kleywegt, Nori, and Savelsbergh, 2002).

\section{PROBLEM STATEMENT}

IRP is concerned with the repeated distribution of a single product, from a single facility, i.e. a central depot, to a set of $N=\{1,2, \ldots, n\}$ dispersed retailers over a given planning horizon of length $T$. For each retailer $i \in N$, we have a demand $d_{i}^{t}$, and a level of inventory $I_{i}^{t}$ (with a maximum of $C_{i}$ ) per time period $t \in \mathrm{T}$. The amount of delivery $q_{i}^{t}$, to customer $i$ in period $t$, is to be decided and based on the delivery amounts in period t. Each retailer $i$, incurs an inventory holding cost of $h_{i}$ per period per unit. We assume that the depot has a sufficient supply of items to cover all customers' demands throughout the 
planning horizon. Deliveries can be carried out at any time period $t \in \mathrm{T}$, using vehicles with virtually unlimited capacity. Any combination of retailers can be visited in a single delivery route and the transportation $\operatorname{cost} c_{i j}$, i.e. from retailer $i$ to retailer $j$, is given. The total cost comprises of the inventory holding cost and the transportation cost, as depicted in the objective function (1).

$$
\min \sum \sum \boldsymbol{h}_{i}{ }^{t}+\sum \sum \sum \boldsymbol{c}_{i j} \boldsymbol{w}_{i j}{ }^{t}
$$

subject to the following constraints:

$$
\begin{aligned}
& I_{i}^{t+1}=I_{i}^{t}+q_{i}^{t}-d_{i}^{t} \\
& I_{i}^{t} \leq C_{i} \\
& \sum_{j} w_{i j}^{t} \leq 1, \quad \forall i, t \\
& z_{i j}^{t} \leq M w_{i j}^{t} \quad \forall i, j, t \\
& \sum_{i j}^{t} w_{i j}^{t} w_{l i}^{t}=0 \\
& \sum_{l} z_{l i}^{t}-\sum_{k} z_{i k}^{t}=q_{i}^{t} \quad \forall i, t \\
& I_{i}^{t}, q_{i}^{t}, z_{i j}^{t} \geq 0, w_{i j}^{t}=0,1, \mathrm{M}: \text { large number }
\end{aligned}
$$

Constraint (2) is the inventory balance equation for the customers. Constraint (3) limits the total amount of inventory to $C_{i}$. Constraint (4) ensures that a vehicle will not visit the location of a specific retailer more than once. $w_{i j}^{t}$ is a binary variable that is equal to 1 , if the vehicle visits retailers at location $i$ and $j$ successively. Constraint (5) makes sure that if there isn't a vehicle travelling between two locations (i.e. retailers) then the amount delivered between them will be zero. $z_{i k}^{t}$ is a continuous variable representing material flow. Constraint (6) is used in order to ensure route continuity and constraint (7) reflects the equity between materials flow and the quantity of products to be delivered. Constraints (8) are domain constraints.

\section{GENETIC ALGORITHMS}

Genetic algorithms (GAs) are efficient heuristic procedures often surpassing the effectiveness of more "traditional" algorithms. There are several reasons for that. Firstly, GAs work with a coding of the parameters whereas "normal" optimisation and search procedures use the parameters themselves. Secondly, GAs perform their search from a population of points, not a single point. Thirdly, they use payoff (objective function) information and do not rely on derivatives or other auxiliary information. Finally, GAs make use of probabilistic transition rules, not deterministic ones.

In genetic algorithms, the term "chromosome" typically refers to a candidate solution to a problem, often encoded as a bit string. The "genes" are either single bits or short blocks of adjacent bits that encode a particular element of the candidate solution (e.g., in the context of multi-parameter function optimization the bits encoding a particular parameter might be considered to be a gene). An allele of a bit string is either 0 or 1 ; for larger alphabets more alleles are possible at each locus. In correspondence to the term genotype used in natural systems, in artificial systems the term structure is employed in order to refer to the total package of strings. Moreover, the term "phenotype" is analogous to a "parameter set", or a "solution alternative" that is formed by the decoding of a structure and the locus of a gene is analogous to the position of a bit in a string.

\section{Genetic Operators}


As mentioned earlier, GAs resemble natural systems and basically follow the same principles. In GAs, during each successive generation, a proportion of the existing population is selected to breed a new generation. Individual solutions (strings) are selected through a fitness-based process. In GAs, as a number of genetic operators is employed (selection, crossover, mutation) to ensure that the average fitness of the population of strings will continue to increase in successive generations, good partial solutions combine to form even better composite solutions.

Genetic operators lie at the core of GAs and are of crucial importance to the ability of the algorithm to produce high quality solutions. Selection ensures the survival of the fittest and resembles the "natural selection" process. It determines during each successive generation, which strings will reproduce and pass on their genes to the next generation, according to fitness criteria. Popular and well-studied selection methods include roulette wheel selection and tournament selection. In roulettewheel selection, the fitness function assigns a fitness value to possible solutions (strings).

After selecting the strings, the next step is to generate a new population of solutions from those selected through genetic operators: crossover (also called recombination), and/or mutation. For each new solution to be produced, a pair of "parent" solutions is selected for breeding from the pool selected previously. By producing a "child" solution using the methods of crossover and mutation, a new solution is created which typically shares many of the characteristics of its "parents". New parents are selected for each child, and the process continues until a new population of solutions of appropriate size is generated. These processes ultimately result in the next generation population of chromosomes that is different from the initial generation. Generally the average fitness will have increased by this procedure for the population, since only the best organisms from the first generation are selected for breeding, along with a small proportion of less fit solutions, for reasons already mentioned above.

Analytically, during the process of crossover, two individuals are selected from the population and a crossover site (a position) along the bit strings is randomly chosen. Then, substrings from corresponding positions within the individuals are exchanged. One or both of the new individuals are inserted into the population at the next generation. For example if $\mathrm{S}_{1}=000000$ and $\mathrm{S}_{2}=111111$ are the parent strings and the crossover point is 2 then $\mathrm{S}_{1}{ }^{\prime}=110000$ and $\mathrm{S}_{2}{ }^{\prime}=001111$. Mutation helps the GA procedure to maintain genetic diversity from one generation of a population of chromosomes to the next. A common method of implementing the mutation operator involves generating a random variable for each bit in a sequence. This random variable tells whether or not a particular bit will be modified

\section{Structure of the Algorithm}

The steps for implementing a GA are the following:

1. Randomly create an initial population (generation 0 )

2. Iteratively perform the following sub-steps on the population until the termination criterion (i.e. a satisfactory fitness level or a maximum number of generations), is satisfied:

a. $\quad$ For each string in the population calculate its fitness.

b. Select one or two individual(s) from the population with a probability based on fitness to participate in the genetic operations in (c).

c. Create new individual for the population by applying the following genetic operations with specified probabilities:

$\checkmark \quad$ Reproduction: Copy the selected individual to the new population.

$\checkmark \quad$ Crossover: Create new offspring(s) for the new population by recombining randomly chosen parts from two individuals.

$\checkmark \quad$ Mutation: Create one new offspring for the new population by randomly mutating a randomly chosen part of one selected individual.

3. After the termination criterion is satisfied, the single best individual (string) in the population produced during the run (the best-so-far individual) is harvested and designated as the result of the run. If the run is successful, the result may be a solution to the problem.

\section{A Genetic Algorithm Adapted to the Inventory Routing Problem}

In order to use GAs for solving a problem, the encoding of candidate solutions is necessary . Refering to IRP, a representation of a solution is a two-dimensional matrix (Abdelmaguid and Dessouky, 2006), with each cell containing the quantity of product delivered to customer $i$ during time period $t$, each row corresponding to a specific customer I and each column corresponding to a time period $t$. It is worth noting that delivery amounts are set to be integers. 
An initial population is required. The genesis of this population is conducted using a two-step heuristic algorithm. In phase one, the heuristic procedure examines which customers are in need of a delivery. Generally, retailers are served if their inventory level in period $t$ cannot cope with the demand of that period. Firstly the heuristic decides which customers are in immediate need of a delivery, simply by using a function that calculates the inventory level of customer $i$, in period $t$. In phase two, a network of the customers who are to be serviced is generated and the transportation cost, for supplying each retailer in the network is minimised with the use of a modified Dijkstra algorithm. The amount of product to deliver to each retailer in the network is $q_{i}^{t}=d_{i}^{t}-I_{i}^{t-1}$. This procedure is repeated for each of the time periods. The heuristic also makes sure that none of the constrains of the IRP is violated by the proposed solution.

\section{Genetic Operators}

The selection of individuals to produce successive generations plays an extremely important role in GAs. The basic methods we have chosen to apply are selection based on roulette wheel and elitism. Our selection operation at first locates the elite chromosome that is the solution with the least total cost. This solution is automatically passed on to the new population in order to safeguard that it will not be "eliminated" during the selection process. Next, the fitness of each individual is computed. As we are dealing with a minimization problem in that our goal is to minimize the sum of the inventory and routing costs for a number of periods, it is vital to map the underlying natural objective function to a fitness function. This transformation is performed using the method proposed by Goldberg (1989), according to which:

$f(x)=C_{\text {max }}-g(x)$ when $g(x)<C_{\max }$

$f(x)=0 \quad$ otherwise

where $g(x)$ is the cost minimization function, $f(x)$ is the fitness function and $C_{\max }$ the largest $g$ value in the current population. The new population is then created using the fitness values computed earlier and a roulette wheel mechanism.

Given the nature of the problem and the encoding selected, crossover is the most intriguing operator. Crossover in our case can be performed either horizontally or vertically. While horizontal crossover, that is random exchange of delivery schedules (rows) of certain customers between the "parent" solutions, poses no problem, vertical crossover may cause a violation of the constraint concerning the capacity of each retailer or the non-stock out policy. Vertical crossover i.e. exchange of rows between "parent" solutions corresponds to the possibility of having excessive amounts of product which in turn results to poor fitness -as inventory holding cost are too great- or violation of the constraints of the problem. Consequently, the latter method is not given any possibility to occur.

Following the crossover operation, mutation is applied to each offspring generated with a probability equal to the mutation rate. Specifically the amount of product to be delivered is mutated (decreased or increased) randomly by a small amount; and consequently new solutions are created. As with the previous operators, violations of constraints by the mutated solutions are not allowed.

\section{ILLUSTRATIVE EXAMPLE}

The proposed method is applied to a simple example which concerns a single depot and six customers. The distribution plan spans over 3 periods, while demand, holding costs, and distances between customers and depot are shown in Tables 1-3 respectively. Beginning inventory at $\mathrm{T}=0$ is also presented in Table 3. Maximum inventory capacity for each customer is 29 , $40,19,42,94,32$ respectively.

Results computed for the distribution plan are presented in Table 4, whilst the corresponding routes travelled in each time

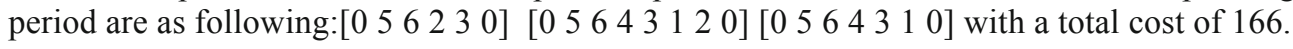

\section{CONCLUDING REMARKS}

The purpose of this work is to present a GA approach for solving the Inventory Routing Problem. The paper consists of three parts. Firstly, IRPis introduced, followed by a formal problem statement. A description of the problem, using a model of Mixed-Integer Programming is given at the of this part. Secondly, the basic theoretical foundations for Genetic Algorithms are presented and a solution for the Inventory Routing Problem is proposed by solving an illustrative example. 


\section{TABLES}

Table 1: Distances between individual customers and Depot

\begin{tabular}{|l|l|l|l|l|l|l|l|}
\hline & 1 & 2 & 3 & 4 & 5 & 6 & $\mathrm{D}$ \\
\hline 1 & 0 & 5 & 1 & 30 & 10 & 4 & 5 \\
\hline 2 & 5 & 0 & 4 & 15 & 20 & 18 & 32 \\
\hline 3 & 1 & 4 & 0 & 9 & 10 & 25 & 6 \\
\hline 4 & 30 & 15 & 9 & 0 & 15 & 3 & 10 \\
\hline 5 & 10 & 20 & 10 & 15 & 0 & 9 & 1 \\
\hline 6 & 4 & 18 & 25 & 3 & 9 & 0 & 2 \\
\hline D & 5 & 32 & 6 & 10 & 1 & 2 & 0 \\
\hline
\end{tabular}

Table 3: Demand per period $\left(\boldsymbol{d}_{\boldsymbol{i}}{ }^{t}\right)$ and beginning inventory $\left(I_{i}{ }^{0}\right)$ for each customer i

\begin{tabular}{|l|l|l|l|l|}
\hline & $\left(\boldsymbol{d}_{\boldsymbol{i}}^{\mathbf{1}}\right)$ & $\left(\boldsymbol{d}_{\boldsymbol{i}}^{\mathbf{2}}\right)$ & $\left(\boldsymbol{d}_{\boldsymbol{i}}^{\mathbf{3}}\right)$ & $\left(\boldsymbol{I}_{\boldsymbol{i}}{ }^{\mathbf{0}}\right)$ \\
\hline 1 & 10 & 11 & 12 & 12 \\
\hline 2 & 15 & 15 & 8 & 14 \\
\hline 3 & 12 & 10 & 11 & 2 \\
\hline 4 & 13 & 10 & 9 & 14 \\
\hline 5 & 14 & 13 & 7 & 9 \\
\hline 6 & 8 & 14 & 15 & 1 \\
\hline
\end{tabular}

Table 4: Distribution plan for each customer per period

\section{REFERENCES}

Abdelmaguid, T.F. and Dessouky M.M. 2006. "A genetic algorithm to the intergrated invrntory-distribution problem". International Journal of Production Research 44 : 4445-4464.

Anily S. and Bramel J. 2004. “A Probabilistic Analysis of a Fixed Partition Policy for the Inventory-Routing Problem”. Naval Research Logistics 51: 925-948.

Bard, J., L. Huang, P. Jaillet and M. Dror.A. 1998. "Decomposition Approach to the Inventory Routing Problem with Satellite Facilities". Transportation Science 32: 189-203.

Bertazzi, L, Paletta, G and Speranza, M. 2002. "Deterministic order-up-to level policies in an inventory routing problem". Transportation Science 36 : 119-132.

Bramel J and Simchi-Levi D. 1997. The Logic of Logistics. Springer: New York.

Campbell A., Savelsbergh M.W.P. 2004. “A decomposition approach for the inventory-routing problem”. Transportation Science 38 : 488-502.

Chien, W., Balakrishnan, A. and Wong, R. 1989 “An integrated inventory allocation and vehicle routing problem”.

Transportation Science 23 : 67-76

Goldberg D. 1989. Genetic Algorithms in Search, Optimization, and Machine Learning, Addison-Wesley Professional. 
Kleywegt J., Nori V. S., and Savelsbergh M. W. P. 2002. "The Stochastic Inventory Routing Problem with Direct Deliveries". Transportation Science 36 : 94-118. 